\title{
Fuel rod nonlinear vibrations to detect and characterize Pellet-Cladding Interaction
}

\author{
V. D’Ambrosi ${ }^{\mathrm{a}, *}$, C. Destouches ${ }^{\mathrm{a}}$, G. Ricciardi ${ }^{\mathrm{b}}$, S. Breaud ${ }^{\mathrm{a}}$, F. Lebon ${ }^{\mathrm{c}}$, J.M. Gatt ${ }^{\mathrm{d}}$, J. Julien ${ }^{\mathrm{d}}$, D. Parrat ${ }^{\mathrm{d}}$ \\ ${ }^{\text {a } C E A, ~ D E S, ~ I R E S N E, ~ D E R, ~ I n s t r u m e n t a t i o n ~ S e n s o r s ~ a n d ~ D o s i m e t r y ~ L a b o r a t o r y, ~ C a d a r a c h e, ~ F-13108 ~ S t ~ P a u l ~ L e z ~ D u r a n c e, ~ F r a n c e ~}$ \\ ${ }^{\mathrm{b}}$ CEA, DES, IRESNE, DTN, F- 13108 St-Paul-lez-Durance, France \\ ${ }^{\mathrm{c}}$ Aix Marseille Univ, CNRS, Centrale Marseille, LMA, Marseille, France \\ ${ }^{\mathrm{d}}$ CEA, DES, IRESNE, DEC, F- 13108 St-Paul-lez-Durance, France
}

\begin{abstract}
Pellet-Cladding Interaction (PCI) in a Light Water Reactor is one of the major concerns to guarantee clad integrity while attempting at increasing the flexibility of PWR nuclear reactor operations to follow power grid demand. In order to forbid operations leading to clad failure, modeling capability to simulate the mechanism has improved through the years. Code development needs detailed and precise experimental data. Those data result from dedicated irradiation programs, named "power ramp tests", carried out in experimental devices in Material Testing Reactors (e.g. in France ISABELLE in OSIRIS in the past (Alberman et al., 1997), the ADELINE loop in the Jules Horowitz Reactor JHR in the next future (Cheymol et al., 2011)). Those irradiation devices are highly instrumented to collect the most relevant information with the highest possible accuracy. In parallel with information gained thanks to post-irradiation examination programs, research is working on innovative methodologies to detect and characterize PCI kinetics during the tests. In this frame, we investigate the technological feasibility to measure the effects of PCI, on the vibrations of a nuclear fuel rod externally submitted to the turbulent axial flow rate excitation. We designed and realized the out-of-pile IMPIGRITIA set-up to reproduce, in controlled laboratory conditions, the mechanical interaction originating in the nuclear reactor. A single PWR rod test section, reproducing the main relevant geometrical and material characteristics of ADELINE experimental system, is submitted to the hydraulic excitation representative of the real case. The local closure of the gap is obtained by means of a remotely controlled expansion system. Measurements of the transverse displacement of the sample rod are collected by Laser Doppler Vibrometry. In this paper we introduce the design of the mock-up and the associated measurement method, then we present the two experimental phases and their results: the first one in air, to show the feasibility of the measurement in air in controlled conditions; the second one under turbulent flow rate to state on the feasibility of the passive detection. At the end, conclusions and perspectives for the work are discussed.
\end{abstract}

\section{Introduction}

Pellet-Cladding Interaction (PCI) constitutes an issue in the definition of technical specifications for the operation of nuclear power plants and for the identification of associated safety margins. During the normal operation of a nuclear Pressurized Water Reactor (PWR) inside the fuel rod, pellets expand and clad creeps inward. PCI establishes and mechanical stresses are induced in the clad. Due to the axial power profile and temperature field, this phenomenon first origins at the mid- plane of the fuel rod and then propagates towards its bottom ends. Stresses origin from the interaction. They are low in normal operations but rise in the case of power transient, start-up and control rod maneuvering operations followed by low thermal range. The cladding prevents the release of radioactive fission products in the primary loop of the reactor and the French Nuclear Safety Authority demands that its integrity is ensured during normal and class II transient operations (https://www.asn.fr/Professionnels/Installations-nucleaires/Guidesde-1-ASN/Guides-de-1-ASN-n-22-Conception-des-reacteurs-a-eau-sous-

\footnotetext{
* Corresponding author.

E-mail address: veronica.dambrosi@cea.fr (V. D’Ambrosi).
} 
pression, online version, 18th July, 2017).

Modeling is a fundamental tool to understand and drive the definition of experiences to better understand the mechanism. Among the available simulation platforms, Alcyone of the PLEIADES platform is a reference tool to simulate fuel rod thermal-mechanical behavior in normal and off-normal operations (Michel et al., 2013). In order to improve the knowledge of PCI kinetics during irradiation, an on-line measurement method designed to detect and characterize all the relevant parameters is being developed. The objective of the measurement set-up is to instrument the test section of the future ADELINE loop in the JHR. The device shall be dedicated to simulate transient irradiation sequences under PWR temperature and pressure conditions. Innovative solutions are being studied to detect and characterize the PCI mechanism, and access new information to improve modeling capability.

In this frame, we studied the technological feasibility to analyze rod vibrations to detect the origin and evolution of the pellet-cladding contact. The objective is to state on the experimental feasibility to detect the pellet-cladding contact by the analysis of rod vibrations. We present in the following Section 2 a review of the state of the art on the topic. Then, we proceed in Section 3 to present the design of the test bench IMPIGRITIA and the measurement method. This part was presented in extended way in (D'Ambrosi et al., 2019). The system has been designed to be representative of the geometry of ADELINE irradiation device and to recreate a representative turbulent axial excitation. In Section 4 we present the experimental program which was performed in two phases. The first was carried out in air to state the feasibility to detect the contact under laboratory conditions. The experimental evidence of a change in the dynamic behavior of the rod depending on the pellet-cladding local contact supported the realization of the second phase of the experimental campaign. This was performed under axial turbulent excitation, to study the feasibility of the detection under Flow Induced Vibrations (FIV) and the impact of flow rate on the feasibility of the detection. After presenting the methodology for data treatment and analysis, Section 5 is devoted to the discussion of results. Conclusions and further work are presented in Section 6.

\section{State of the art}

When a structure is submitted to an axial turbulent fluid flow rate, FIV origin from the interaction of the structure with the fluid. According to (Païdoussis, 2004; Paidoussis, 1981) and Axisa (Axisa, 2001), the mechanisms exciting the system undergoing FIV are twofold: the near field components and the far field ones. The near field components are pressure fluctuations imposed by the fluid due to different contributors but the drag force in the boundary layer turbulence is the most important one. The far field components are due to all systems and loop dependent noises which propagate in the loop at the speed of sound (for example flow pulsations, vortex shedding, turbulence generated by bends, cavitation, valves). This implies that the external force soliciting the fuel rod and the consequent rod vibrations strongly depends on the specific loop configuration.

Information concerning the fluid flow rate are not always easily available because of the need to install a dedicated instrumentation. Semi-empirical correlations have been developed to estimate the amplitude of transverse vibrations of simply supported rods submitted to the turbulent excitation induced by the axial flow. These are characterized in terms of the root mean square amplitude $y$ divided by the characteristic dimension for the rod, its diameter $D_{c}$. We can mention the correlations proposed by Paidoussis (1969), Chen and Wever (1970) and Chen and Wambsganss (1971). According to Païdoussis (2004) transverse vibrations rarely exceed a ratio $\mathrm{y} / \mathrm{D} \sim 10^{-3}$. In the considered case of nuclear fuel rods, the rod diameter is about $1 \mathrm{~cm}$ which corresponds to a an expected displacement amplitude in the order of the $\mu \mathrm{m}$.

As reported by Chen in Chen and Wambsganss (1985), the vibrations of cylindrical structures submitted to the near field flow noise are characterized by two aspects: (i) the system responds according to its first mode, while the contributions due to higher modes are limited at low flow velocities; (ii) the excitation mechanism is random and generally expressed in terms of a power spectral density.

Scientific literature in the nuclear context has mainly focused on the fretting phenomenon which occurs at the interface between fuel rods and spacer grids as a consequence of FIV. Few researches have been carried out on the impact of the non linear dissipation mechanisms which take place inside the fuel rod, due to the presence of pellets and the existence of a fabrication gap between pellets and gap (Catterou et al., 2020). When the gap is open and FIV are induced, a relative motion establishes between the cladding and the fuel pellets. This relative motion origins pellet-pellet friction and pellet-cladding shock in the transverse direction. Due to the recognized difficulty in correctly modeling those phenomena (Ferrari and Balasubramanian, 2018), these mechanisms are usually approximated. Benhamadouche in (Benhamadouche et al., 2009) uses a linear approximation method, by evaluating an equivalent mass density for the rod which takes into account pellet mass density. The fuel rod is then studied as an Euler-Bernoulli beam. Park in Park et al. (2009) studies the fuel rod as a linear system but makes the point that due to the presence of the gap, the contribution of the mass of pellets to the density of the global structure cannot be clearly determined. He proposes to compare the natural frequencies of the empty rod and the fulfilled one to estimate the appropriate density. None of these approaches allows to study the effect of gap local closure and its progressive closure along the axial length of the rod.

Ferrari recently proposed an experimental characterization to study the non linear vibrations of nuclear fuel rod, in air and in quiescent water (Ferrari and Balasubramanian, 2018). In this study, he considered a $900 \mathrm{~mm}$ zircaloy clad, clamped at both extremities, eventually fulfilled with cylindrical tungsten carbide pellets, similar to uranium fuel pellets in terms of dimensions and density. He compared the dynamics of an empty rod, a fulfilled rod freely moving (no spring at one bottom of the fuel column) and a fulfilled rod axially constrained in air and in quiescent water. From measurements he observed that pellets decrease natural frequencies with respect to the empty rod, and that axial constriction increases natural frequencies and reduces damping with respect to freely moving configuration, both in air and in water.

In this study we focused on non linear dissipation mechanisms on the dynamics of the fuel rod and on the influence of the pellet-cladding contact on these mechanisms.

\section{Design and measurement method}

Mechanical dissipation, as mentioned, arises from the presence of pellets inside the clad and the relative motions between them and the cladding (Ferrari and Balasubramanian, 2018) when the radial gap is open. In a generic PWR rod, the radial gap size is about $100 \mu \mathrm{m}$. The presence of the pellet-cladding gap and the excitation of the sample rod induced by the flow induced vibrations, origin a relative motion between the two components of the sample rod and the appearance of pellet-cladding shocks and pellet-pellet friction, that are dissipation mechanisms. These mechanisms contribute to the total energy dissipated in the structure and damp the system vibrations.

The pellet-cladding contact reduces the relative motion, with the consequent reduction of the dissipated energy. This can result into a change of the system frequencies, a modification of the viscous damping ratio of the structure and, for a system submitted to forced excitation, a variation of vibrations amplitude for a fixed external excitation.

\subsection{The design}

The application frame for the study is the ADELINE irradiation loop of the JHR research reactor. According to the present design, the ADELINE in-pile part is a $4 \mathrm{~m}$ height device, with a single rod test section where PWR thermal and pressure conditions are reproduced (155 bar 
and $275^{\circ} \mathrm{C}$ inlet water temperature) (Gonnier et al., 2017). The fuel rods to be tested in ADELINE are obtained from the $4 \mathrm{~m}$ original PWR rods according to a qualified refabrication process and have a length of about $50 \mathrm{~cm}$ (Alberman et al., 1997). The refabricated fuel rod to be tested is held in the section by a sample holder for a total length of about $4 \mathrm{~m}$ (sample holder + fuel rod) and the structure is centered in the test section by means of four centering elements. The fuel rod is cooled during the test by means of an up-streaming turbulent flow rate.

The design criteria considered for IMPIGRITIA are the environmental conditions, the geometrical design and the fixation conditions, the material characteristics and the hydraulic excitation mechanism.

\subsubsection{The environment}

For this feasibility study, IMPIGRITIA mock-up was designed to work at low pressure and controlled room temperature. The high pressures and temperatures of the ADELINE device as well as the neutron irradiation, modify the thermal-mechanical properties of the system with respect to room temperature. Theoretical and experimental correlations are available to describe the evolution of properties with the environment (Fink, YYYY) and allow in the post-processing phase to establish the expected behavior of the system in the ADELINE conditions.

\subsubsection{The hydraulic design}

Hydraulics is designed in order to reproduce the external solicitation in terms of the axial turbulent flow and pressure fluctuation in the flow. We identified a range of representative flow rates, imposing two principles: the conservation of wall pressure fluctuations and the Burgreen principle (Prakash et al., 2011). The conservation of wall pressure fluctuation $p_{r m s}$ was imposed according to the definition proposed by Axisa in Axisa (2001) on the basis of the envelop PSD derived from Clinch data (Clinch, 1969) and reported in Eq. (1).

$p_{r m s}=0.003\left(\rho v^{2}\right)$

where $p_{r m s}$ is the root mean square of pressure fluctuations, $\rho$ is fluid density and $v$ is the average flow velocity.

The criterion established by Burgreen has been recently used by De Pauw et al. (2015) to study flow induced vibrations in a prototype representative of the MHYRRA system and it is reported in Eq. (2).

$v_{\text {exp }}=v \frac{\rho^{0.5} \mu^{-0.33} E^{-0.33} M^{0.165}}{\rho_{\text {exp }}^{0.5} \mu_{\text {exp }}^{-0.33} E_{\text {exp }}^{-0.33} M_{\text {exp }}^{0.165}}$

Subscript 'exp' stands for prototype, $\mu$ the fluid dynamic viscosity, $E$ elastic modulus and $M$ the mass of the sample rod at operating temperature.

Applying the described criteria in our test conditions we defined a range of representative flow rates. Fluid flow velocity ranges between 0.9 to 2.6 the fluid velocity in the hydraulic channel surrounding the sample rod in ADELINE.

$\frac{v_{\text {exp }}}{v} \in[0.9: 2.6]$

\subsubsection{The geometry and the fixation conditions}

The geometry and the fixation conditions strictly impact the dynamics of a structure and its response under flow rate excitation. Fixation conditions of ADELINE sample is thus maintained in the experimental set-up: the sample holder is fixed only at the top of the experimental section and it is centered by means of 4 annular elements. A simplification in the geometrical configuration has been carried out, as detailed in D'Ambrosi et al. (2019). IMPIGRITIA is indeed shorter than ADELINE. For exploitation issues, we limited our study to the lower part of ADELINE sample holder, where the sample rod is located. The resulting experimental device has a length of about $1.5 \mathrm{~m}$.

Two centering elements are placed towards the top of the sample holder and two towards the bottom. The minimum radial gap between the rod and the internal diameter of the centering element amounts to
Table 1

Eigen-frequencies of ADELINE and IMPIGRITIA devices by linear approximation. Frequencies are reported in $\mathrm{Hz}$.

\begin{tabular}{lcccc}
\hline Device & \multicolumn{4}{c}{ Frequency [Hz] } \\
\cline { 2 - 5 } & $f_{1, l}$ & $f_{2, l}$ & $f_{3, l}$ & $f_{4, l}$ \\
\hline ADELINE & 1.5 & 7.6 & 13.0 & 21.6 \\
IMPIGRITIA & 6.2 & 29.0 & 79.9 & 170.2 \\
\hline
\end{tabular}

$250 \mu \mathrm{m}$.

The shorter sample holder has two main consequences on the feasibility study: on one side, a shorter system strengthens the relative impact of a local pellet-cladding interaction on the global dynamics of the structure, thus enhancing the possibility to measure the impact of the local contact. On the other side, it increases the system stiffness which result in smaller vibration amplitudes for the same excitation mechanisms, making it more difficult to measure the researched phenomenon.

The modifications in the configuration of the system increase the eigen-frequencies of the designed system with respect to ADELINE. Table 1 summarizes the first four expected eigen-frequencies $f_{i, l}$ for ADELINE and IMPIGRITIA. These are obtained considering the geometry and material properties of each structure and modeling it as a linear Euler Bernulli cantilevered beam.

The geometrical characteristics of the fuel rod were defined according to the FABRICE type fuel rods to be used in ADELINE: zirconium alloy claddings were supplied, having an external diameter equal to 9.5 $\mathrm{mm}$, an internal one of $8.36 \mathrm{~mm}$ and a length of $550 \mathrm{~mm}$. We replaced the uranium material constituting the pellets with molybdenum, which is representative of $\mathrm{UO}_{2}$ density. Cylindrical pellets were fabricated, having an external diameter of $8.2 \mathrm{~mm}$. A total number of 33 pellets were located inside the clad, for an equivalent length of the fuel stack of $495 \mathrm{~mm}$.

A synthesis of geometrical and material properties is reported in Table 2 .

Pellets were axially constrained by an inconel spring having the geometrical and material properties of the ones used in PWR fuel rods.

To obtain water-tightness, each side of the cladding tube was welded to a plug. The whole was then screwed to the sample holder, a prolongation tube in stainless steel which was fixed at the top of the experimental section. The other side was free to move.

The radial pellet-cladding gap amounted to $80 \mu \mathrm{m}$. In order to fulfill it and remotely induce the pellet-cladding contact, a dilatation system capable of local expansion is used. It has been developed for stent implantation in the medical domain and it presented a balloon which expanded when inflated. The system could be remotely controlled by a connection tube. A general assembly scheme is presented in Fig. 1: the component was introduced from the top of the sample rod, through centrally drilled pellets and the balloon was placed in the region where gap closure wanted to be induced. There, pellets were replaced by two half pellets, which were displaced by means of the previously described dilatation of the system, leading to pellet-cladding contact.

\subsection{The measurement method}

Vibration data were collected through a Laser Doppler Vibrometer

Table 2

Geometrical characteristics and material properties of interest at $T_{A M B}$.

\begin{tabular}{llll}
\hline & Cladding & \multicolumn{2}{c}{ Pellets } \\
\hline Material & Zyrcaloy & $\mathrm{UO}_{2}$ & Mo \\
Density $\left[\mathrm{kg} / \mathrm{m}^{3}\right]$ & 6570 & 10963 & 10200 \\
Young's Modulus [GPa] & 95 & 194.3 & 320 \\
$\mathrm{D}_{\mathrm{e}}[\mathrm{mm}]$ & 9.5 & 8.2 & 8.2 \\
$\mathrm{D}_{\mathrm{i}}[\mathrm{mm}]$ & 8.36 & - & - \\
\hline
\end{tabular}




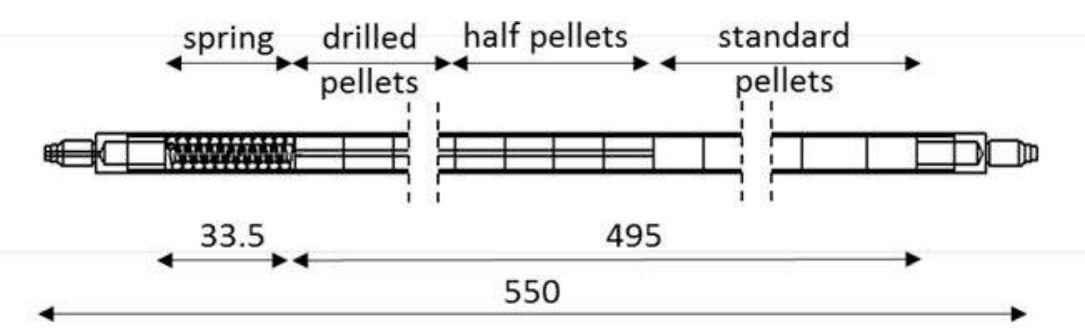

Fig. 1. Fuel rod scheme: in order to allow positioning the dilatation system from the top of the rod to the central region, top pellets are drilled. Where PCI is wanted, pellets are replaced by two half-pellets.

Table 3

Instrumentation installed in IMPIGRITIA test bench and associated main characteristics.

\begin{tabular}{cccc}
\hline Parameter & $\begin{array}{c}\mathrm{f}_{\text {samp }} \\
{[\mathrm{Hz}]}\end{array}$ & Instrument & $\begin{array}{c}\text { Accuracy } \\
(1 \sigma)\end{array}$ \\
\hline Velocity/ & $10^{4}$ & $\begin{array}{c}\text { Laser Doppler } \\
\text { Interfermeter }\end{array}$ & $0.1 \mathrm{~Hz}$ \\
Displacement & & K type thermocouples & $\pm 1.5 \mathrm{~K}$ \\
Temperature & 1 & pressure gauges & $\pm 0.3 \mathrm{bars}$ \\
Pressure & 1 & Vortex effect flow meter & $\pm 21 / \mathrm{min}$ \\
Flow rate & imposed & & \\
\hline
\end{tabular}

(LDV) system: the laser beam was pointed on the vibrating object and it was scattered back from it. The velocity and displacement amplitudes of the studied object generated a frequency or a phase modulation respectively, due to Doppler effect. The laser used was a class 2 Helium-Neon type, having a wavelength of $633 \mathrm{~nm}$ and a cavity length of $204 \pm 1 \mathrm{~mm}$. Located at about $0.5 \mathrm{~m}$ from the moving rod, the spot diameter on the rod was about $50 \mu \mathrm{m}$. The measurements were performed at the bottom of the sample rod, above the bottom centering elements.

The experimental section was completely designed in optical quality PMMA (plexiglass). It was demonstrated by Pauw et al. (2013) that LDV technique leads to a higher signal-to-noise ratio than other measurement techniques as grid method, fiber Bragg grating sensors, electrical strain gauges and accelerometers.

To produce and control flow conditions in the experimental section, we designed a dedicated independent water loop, equipped with a circulation pump, an expansion tank and a cooling system to keep temperature constant in the system during tests. All conditioning components were located on a separate frame to limit noise effect on LDV measures. To monitor conditions in the circuit, we installed a flow meter at the experimental section inlet, three thermocouples located at different positions to verify temperature evolution in the loop and three pressure gauges to check pressure drops. A summary of instrumentation characteristics and accuracy is reported in Table 3. The scheme of the experimental configuration is illustrated in Fig. 2: on the left the configuration for the measurements under flow rate excitation, on the right the one for air tests.

\section{Data acquisition and treatment}

The experimental campaign was organized in two phases: a first one in air and a second one under flow rate. In both, the sample holder is fixed at the top of the experimental section, according to the design of the device. During air tests, the centering elements are removed. Free vibrations tests were performed, imposing an initial displacement to the fixed-free system and exciting the first vibration mode, that is the main one activated under fluid flow rate (Chen and Wambsganss, 1985). The rod presents two possible configurations: open gap (deflated dilatation system) and closed gap (inflated dilatation system).

We collected the displacement and the velocity of the moving rod at a sampling frequency of $10 \mathrm{kHz}$. Measures were repeated $\mathrm{N}$ times for each rod configuration.

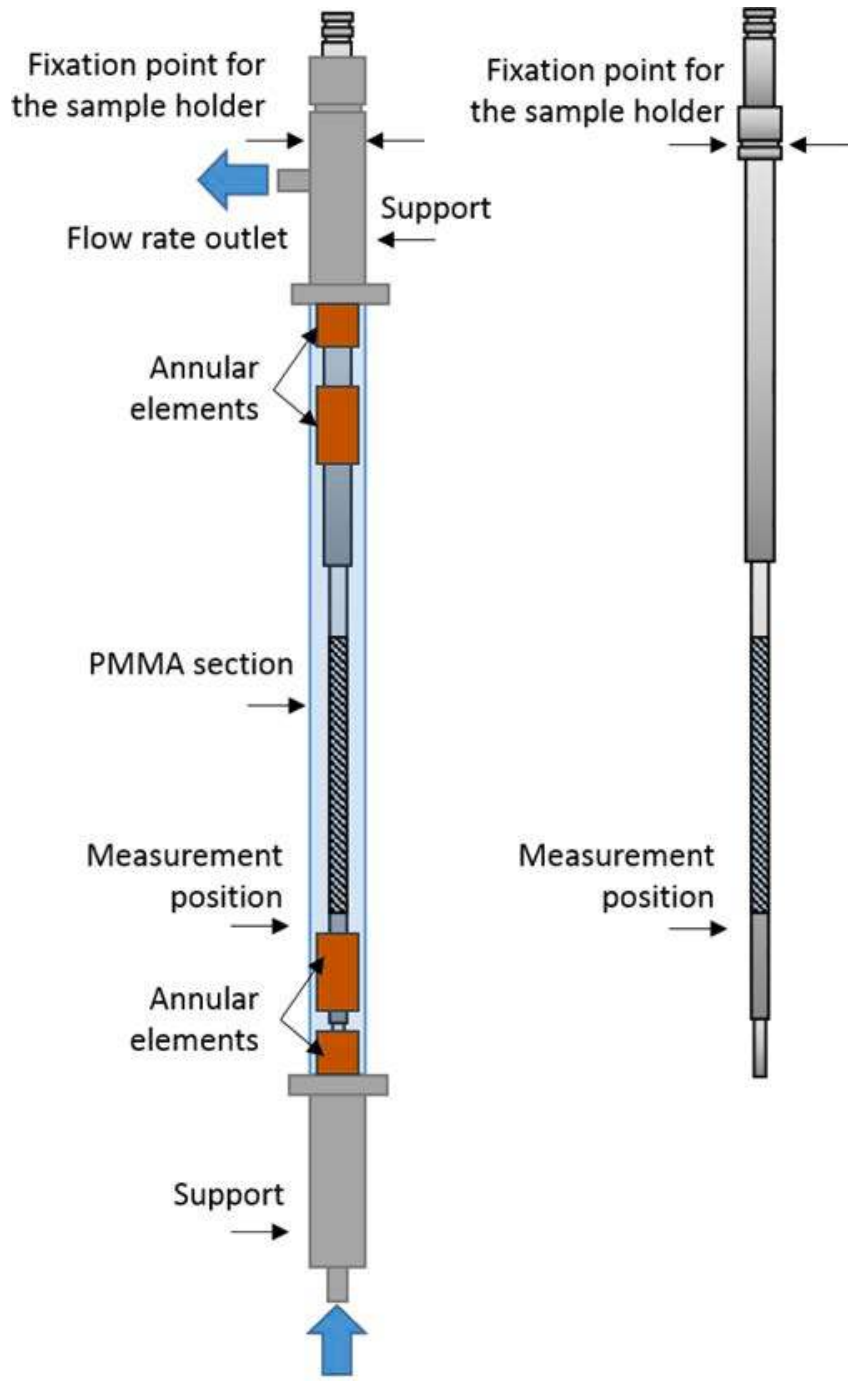

Flow rate inlet

Fig. 2. Schemes of the experimental configurations: on the left the one for flow rate tests, on the right for the free vibrations tests.

Under flow rate excitation, data were collected for a fixed flow rate, in the interval of representative flow rates of the ADELINE device. The excitation mechanism was random, long measures were thus performed to improve the statistics. The sampling frequency was fixed at $10 \mathrm{kHz}$ as for the air measurements. The experiments were performed at four different flow rates within the identified representative interval of ADELINE device: $13.5 \mathrm{l} / \mathrm{min}(0.82 \mathrm{~m} / \mathrm{s}$ at the sample rod), $201 / \mathrm{min}$ $(1.21 \mathrm{~m} / \mathrm{s}), 30 \mathrm{l} / \mathrm{min}(1.82 \mathrm{~m} / \mathrm{s})$ and $41.5 \mathrm{l} / \mathrm{min}(2.5 \mathrm{~m} / \mathrm{s})$.

We have already said that, according to scientific literature (Paidoussis, 1981), the system, submitted to flow induced vibrations, was 
expected to respond according to its first mode. For this, measurements were collected at the bottom of the sample rod, where oscillation amplitudes were the highest.

\subsection{Free vibration tests data treatment}

We applied to the measured signal a low pass Butterworth filter with a cutting frequency of $5 \mathrm{kHz}$. The Consecutive Logarithmic Decrement Method (C-LDM) was used to evaluate the decay of viscous damping ratio of the $i_{\text {th }}$ measurement as a function of the system displacement (Heitz, 2017).

The viscous damping ratio for an under-damped linear Single Degree Of Freedom, SDOF, system can be obtained by Logarithmic Decrement Method, LDM, measuring its displacement along time. The displacement can be written in the following form (Fig. 3):

$x(t)=\exp \left(-\zeta \omega_{0} t\right)\left(\alpha \cos \left(\omega_{d} t\right)+\beta \sin \left(\omega_{d} t\right)\right)$

where $\omega_{0}=2 \pi f_{0}$ is the natural frequency of the SDOF system, $\omega_{d}=$ $\omega_{0} \sqrt{1-\zeta^{2}}$ is the pseudo-angular frequency which is directly measured by the pseudo-period $T_{d}, \alpha$ and $\beta$ depend on the initial conditions of the problem. The term $\exp \left(-\zeta \omega_{0} t\right)$ describes the decay of free oscillations. In this case, to obtain the viscous damping ratio $\zeta$ it is sufficient to consider two different local maxima: considering the peak displacement $x_{i}$ occurring at time $t$ and the peak displacement $x_{i+n}$ at time $t+n T_{d}$ :

$\frac{x_{i}}{x_{i+n}}=\frac{\exp \left(-\zeta \omega_{0} t\right)}{\exp \left(-\zeta \omega_{0}\left(t+n T_{d}\right)\right)}=\exp \left(\zeta \omega_{0} n T_{d}\right)$

For non linear systems the C-LDM, is more adapted: the damping ratio is assumed linear between two successive local maxima and it is evaluated $(N-1)$ times, with $N$ being the number of peak oscillations. The damping ratio results to be a function of the displacement amplitude because of the non linearities in the system. For each test performed, the evolution of the viscous damping ratio $\zeta_{i}(A)$ as a function of the oscillation amplitude was evaluated. We considered a linear relation between the viscous damping ratio and the amplitude of oscillations and we obtained an average evolution $\bar{\zeta}=a+b A$, for each rod configuration. Each average evolution was related to a variance $\sigma_{\bar{\zeta}^{2}}$, resulting from the linear regression of the measured data of each test.

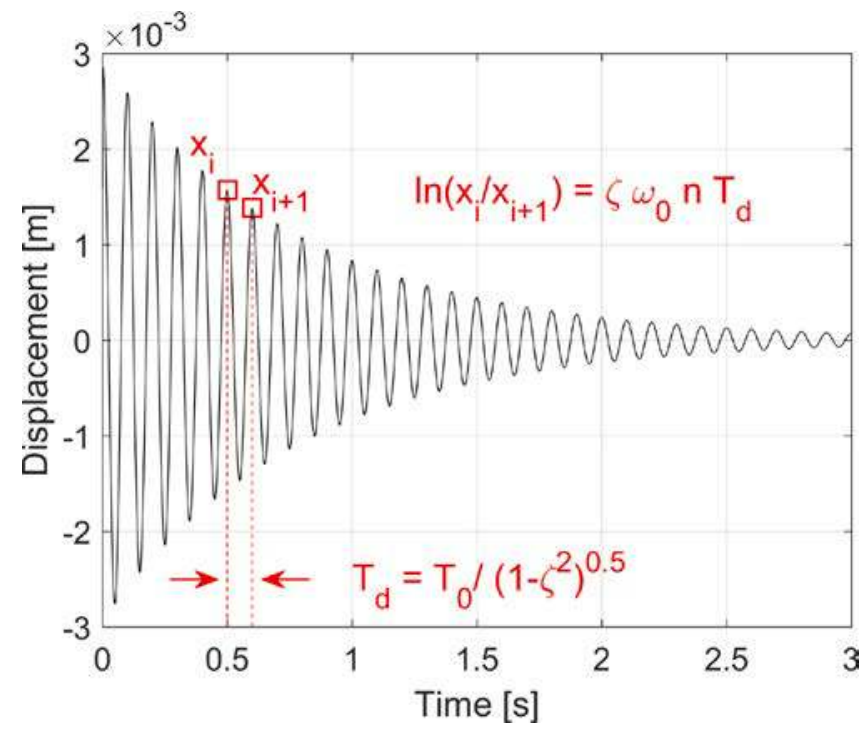

Fig. 3. Principle for the LDM for under-damped free vibrations of a SDOF system.

\subsection{Forced vibrations tests data treatment}

The sample rod velocity of motion in the transverse direction was measured with a typical resolution of $0.5 \mu \mathrm{m} / \mathrm{s}$. The measured vibration direction is kept constant during the whole experiment. The collected signal was noised by the presence of optical dropouts which were removed during the data treatment. An example of the clean signal is proposed in Fig. 4.

Measurements were performed for four different flow rates, chosen within the range of representative excitation mechanisms: $13.51 / \mathrm{min}$ ( $0.8 \mathrm{~m} / \mathrm{s}$ at the sample rod), $20 \mathrm{l} / \mathrm{min}$ ( $1.21 \mathrm{~m} / \mathrm{s}$ at the sample rod), $30 \mathrm{l} /$ $\min (1.82 \mathrm{~m} / \mathrm{s}$ at the sample rod $)$ and $41.5 \mathrm{l} / \mathrm{min}(2.5 \mathrm{~m} / \mathrm{s}$ at the sample rod). The vibrations amplitude of the structure depending on the excitation mechanism is an indicator of the energy dissipated in the system. For a specific value of flow rate, the higher the dissipated energy, the smaller the vibration amplitude is. As reported by Pauw et al. (2013), the absolute value of the vibration amplitude is not a meaningful result because of the random nature of the excitation force. Indeed, for a sufficiently high number of measured samples, the measured displacements of the structure showed to adhere to a Gaussian distribution, according to the central limit theorem. We evaluated the root mean square value of the displacement, which describes the effective displacement of the structure, as follows.

$A=x_{r m s}=\int_{0}^{f_{a}} S_{x x}(f) d f$

where $S_{x x}(f)$ is the power spectral density of the system displacement and $f_{a}$ is the Nyquist frequency.

Each root mean square value of the displacement is related to a variance $\sigma_{A}^{2}$ :

$\sigma_{A}^{2}=\frac{1}{N-1} \sum_{i=1}^{N}\left(x_{i}-A\right)^{2}$

\subsection{Definition of the detection feasibility}

To state on the feasibility of the detection we measured the rod transverse motion when the dilatation system was not inflated (open gap configuration) and compared it to the one measured after inflating the stent and inducing the local pellet-cladding contact (closed gap configuration). No operations were performed on the sample rod between two consecutive tests.

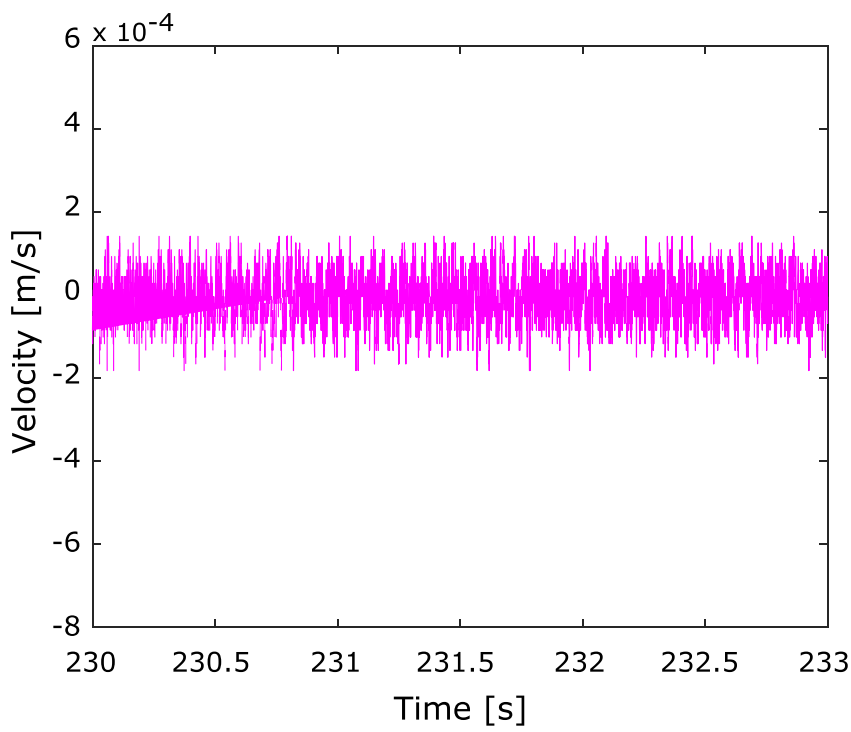

Fig. 4. Example of collected signal during forced vibrations tests after data treatement. 
To characterize the confidence of the detection we evaluated the first order risk $(\alpha)$ and the second order risk $(\beta)$ related to the open gap and closed gap configuration for the same rod under the same excitation mechanism. For the air tests, we took the modeled values of the viscous damping ratio and their variances, for the flow rate ones, the effective amplitude and its variance. We considered each result to be normally distributed. The first order risk for the detection of the pellet-cladding contact is evaluated at the intersection between the open and closed gap measurement and the second order one is consequently deduced.

\section{Results and discussion}

\subsection{Feasibility in air}

Fig. 5 shows the evolution of the damping ratio as a function of the sample rod amplitude of oscillations. The grey curves and the light red curves are the measured evolutions of the $\zeta_{i}(A)$ for each measurement performed, $\mathrm{i}=1 \ldots \mathrm{N}$ where $\mathrm{N}$ is the $\mathrm{N}$ of total tests performed for each rod configuration. In the presented figure, the local closure is induced along about $10 \%$ of the total "fuel" column, thus the $3 / 33$ central pellets. The black and red lines are the modeled linear evolutions and the shaded region corresponds to the relative variance of each modeled evolution, reported at $2 \sigma(\mathrm{k}=2)$. The figure illustrates that the local closure of the gap reduces the damping ratio of the structure in the whole range of oscillations amplitude measured. This agrees with the fact that the local disappearance of the relative motion between the pellets and the cladding results into a reduction of the energy dissipated in the system.

In the range of amplitude oscillations $0.2-1 \mathrm{~mm}$ the detection of the local pellet-cladding contact can be performed with a confidence higher than $95 \%$. The risk of a false detection is $<1 \%$.

For oscillation amplitudes smaller than $0.2 \mathrm{~mm}$ the two modeled evolutions superpose and cannot be statistically distinguished. This is related to a limitation of the measurement method and the experimental procedure: a 1D measurement system is no longer adapted for small amplitudes because of the non linear motion of the structure.

The spectral analysis of the measurements led us to evaluate the first mode frequency of the structure, $f_{1}$. It amounted to $5.7 \mathrm{~Hz} \pm 0.1 \mathrm{~Hz}$. This experimental value is not far from the $6.2 \mathrm{~Hz}$ calculated by a linear approximation of the IMPIGRITIA fuel rod structure and reported in

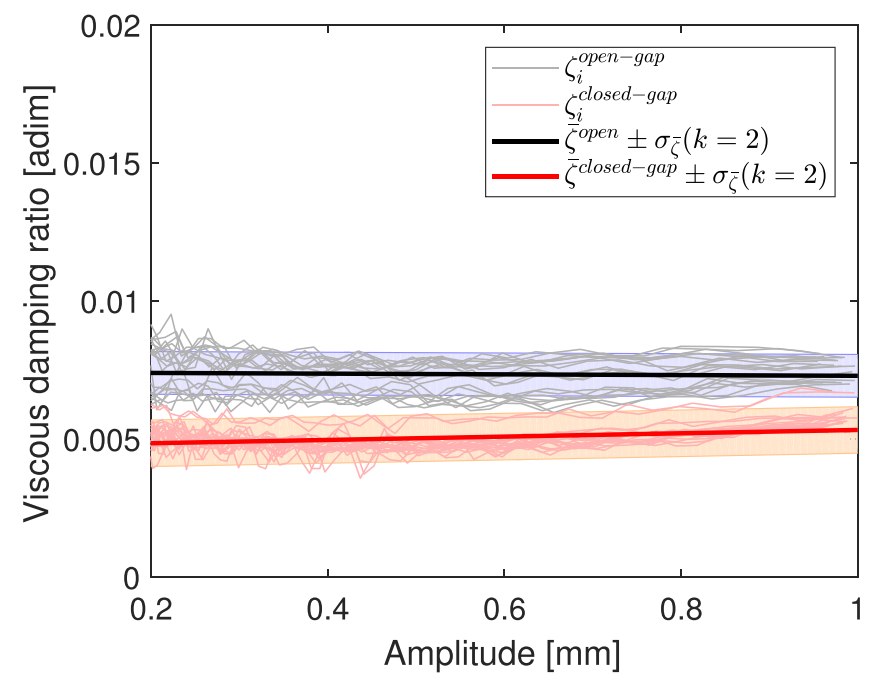

Fig. 5. Viscous damping ratio evolution as a function of the amplitude of oscillations during free rod vibrations. The results are obtained on a sample rod where the gap closure is performed over $3 / 33$ pellets $(10 \%$ of the total fuel column). The $i^{\text {th }}$ evaluated evolutions of $\zeta_{i}(A)$ are represented in light grey for the open gap configuration and in light red for the closed gap one. In black and in red are illustrated the linear model calculated in the range of oscillations, with the associated relative variance $(\mathrm{k}=2)$.
Table 1. From the comparison of the $f_{1}$ of the open and closed gap configuration there was no detectable modification that could be related to the local pellet-cladding contact.

\subsection{Feasibility under passive flow rate excitation}

Fig. 6 and Table 4 present the variation of the vibration amplitude of the structure with the increase of the fluid velocity for the two studied configurations of the sample rod: open gap and closed-gap. The vibration amplitude measured at the bottom of the sample rod was normalized using the outer diameter of the rod, $D_{c}$, equal to $9.5 \mathrm{~mm}$. The variance was reduced coherently with the amplitude. The dimensionless fluid velocity is defined as:

ReducedFluidvelocity $=\frac{u}{D f_{0}}$

where $u$ is the fluid velocity and $f_{0}$ is the natural frequency of the structure.

Values are reported, as before, for the sample rod where the three central pellets were replaced by half-pellets and the dilatation system allows to locally induce the pellet-cladding contact along about the $10 \%$ of the fuel stack. In black are the ones for the open gap configuration and in red for the closed gap one. A zoom is proposed for the three lower flow rate excitation tests.

For reduced fluid velocity lower than 33 , the structure barely moves and it is impossible to identify any modification induced by the local pellet-cladding contact.

For increasing flow rate, the amplitude of oscillations increases because of the increase in the intensity of the turbulence (pressure fluctuations in the boundary layer or near-field noise).

For a reduced fluid velocity equal to 33 , about $60 \%$ of the nominal velocity foreseen in ADELINE, we measured a modification in the effective amplitude induced by the local pellet-cladding contact. From the analysis of the first and second order risk we evaluated that the detection can be performed with a confidence of $90 \%$ (considering unilateral first order risk). The related risk of a false detection $\beta$ amounts to $17 \%$.

At a reduced velocity of 33 , we detected the decrease in the effective amplitude induced by the local closure of the gap and the establishing of the pellet-cladding contact. Despite the expectation on the reduction of the dissipated energy induced by the local closure (as observed in air

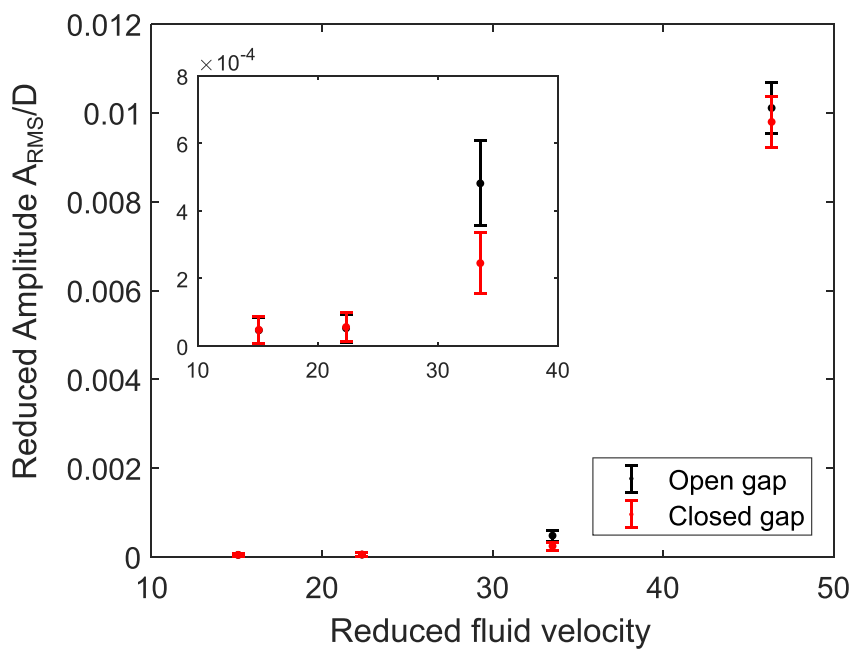

Fig. 6. Evolution of the effective amplitude of the rod oscillation under flow rate excitation. The results are obtained on a sample rod where the gap closure is performed over $3 / 33$ pellets ( $10 \%$ of the total fuel column). In black are the values for the open gap configuration and in red for the closed gap configuration. The variance is illustrated with $\mathrm{k}=1$. 
Table 4

Results of the analysis on the feasibility of the detection from the evaluation of the effective amplitude as a function of the fluid flow rate excitation. The amplitude and its variance are normalized with respect to the diameter of the rod and the velocity using the ratio $u /\left(D f_{0}\right)$.

\begin{tabular}{cccccc}
\hline \multirow{2}{*}{$\begin{array}{c}\text { Reduced } \\
\text { Velocity }\end{array}$} & \multicolumn{2}{c}{ Reduced Amplitude $(\mathrm{k}=1)$} & & \multicolumn{2}{c}{ Risk } \\
\cline { 2 - 3 } \cline { 5 - 6 } & open gap & closed gap & & $\alpha / 2$ & $\beta$ \\
\hline \multirow{2}{*}{15.1} & $4.6 \mathrm{E}-05 \pm$ & $4.8 \mathrm{E}-05 \pm$ & & $100 \%$ & $100 \%$ \\
& $3.9 \mathrm{E}-05$ & $4.2 \mathrm{E}-05$ & & & \\
22.3 & $5.2 \mathrm{E}-05 \pm$ & $5.6 \mathrm{E}-05 \pm$ & & $100 \%$ & $100 \%$ \\
& $1.3 \mathrm{E}-04$ & $5.8 \mathrm{E}-04$ & & & \\
33.5 & $4.8 \mathrm{E}-04 \pm$ & $2.4 \mathrm{E}-04 \pm$ & & $10 \%$ & $17 \%$ \\
& $3.9 \mathrm{E}-05$ & $4.2 \mathrm{E}-05$ & & \\
& $1.0 \mathrm{E}-02 \pm$ & $9.8 \mathrm{E}-03 \pm$ & & $38 \%$ & $40 \%$ \\
& $1.3 \mathrm{E}-04$ & $5.8 \mathrm{E}-04$ & & & \\
\hline
\end{tabular}

tests), the local closure seemed to have the opposite effect during flow rate tests. We retained that this resulted from the configuration of the system and the oscillations amplitudes of the structure. The internal gap between the pellets and the cladding amounted to about $80 \mu \mathrm{m}$. During free vibrations tests on the open gap configuration, the large amplitude oscillations led to the activation of the relative motion between the pellets and the cladding, with the activation of pellet-pellet friction and pellet-cladding shocks. During the flow rate tests performed at a reduced velocity of 33, the effective amplitude of the structure was one order of magnitude lower than the gap width, thus the pellets barely moved inside the cladding. Consequent to the inflation of the system, some energy was dissipated in the contact, resulting into a global decrease of the amplitude oscillations.

For higher imposed flow rate test, amounting to a reduced velocity of 46 corresponding to $100 \%$ of the nominal velocity foreseen in ADELINE (according to the hydraulic similarity described in Section 3), the amplitude of oscillations exponentially increases and the local pelletcladding closure cannot be detected. This highlighted that the feasibility of the measurement is sensitive to the external force exciting the system.

The increase of the structure oscillations amplitude was found in all the other sample rods, submitted to the same flow rates. Fig. 7 presents the evolution of oscillations amplitudes for three tested rods: C1 was a standard rod, with no dilatation system neither modifications of the 33 cylindrical pellets; C3 was the rod where the local pellet-cladding contact can be induced on the three central pellets, previously discussed; C6 was a full-gap rod.

The behavior of the rods at high flow rate was related to the fluid-structure interaction and the presence of the centering elements. Fig. 8 shows the evolution of the power spectral density of the C3 rod as a function of the four applied soliciting forces. The increase of the external force resulted into a strong modification of the structure damping and into a frequency shift. The evolution of modes can be related to the appearance of an instability (Païdoussis, 2004) and/or to a modification of boundary conditions. With the increase of the excitation force, the sample rod interacted with the centering elements. We recall that, by design, a radial gap of about $250 \mu \mathrm{m}$ existed between the sample holder and the annular elements that helped centering the structure in the section. The gap was thus in the same order of the effective amplitude under reduced fluid velocity equal to 46 . The shocks that formed from

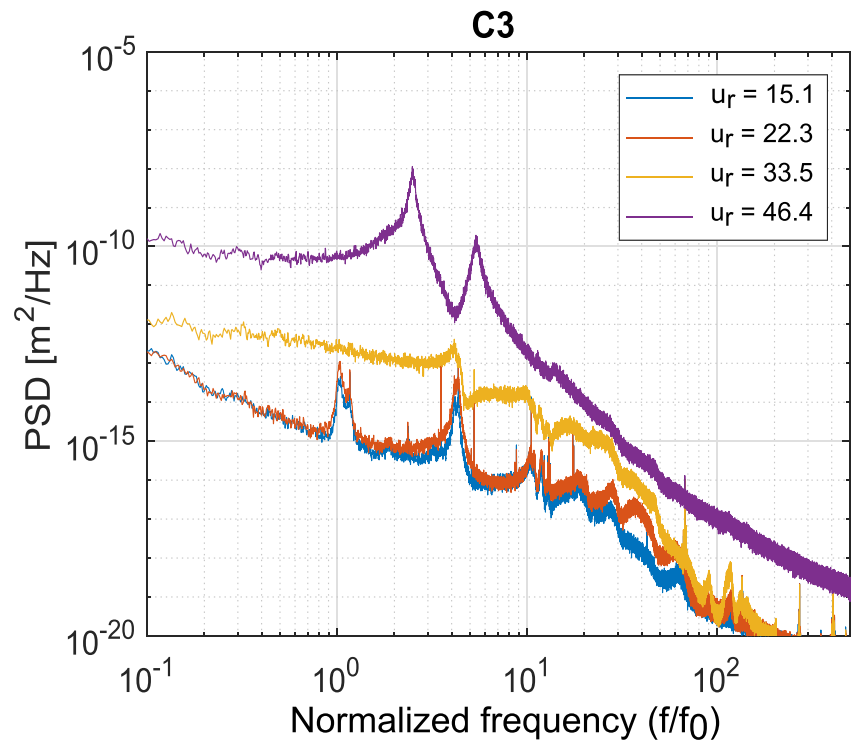

Fig. 8. Evolution of the PSD with the increase of the external force. The appearance of an instability and the chocs of the beam with the annular elements centering the sample rod in the test section lead to a frequency and damping shift.
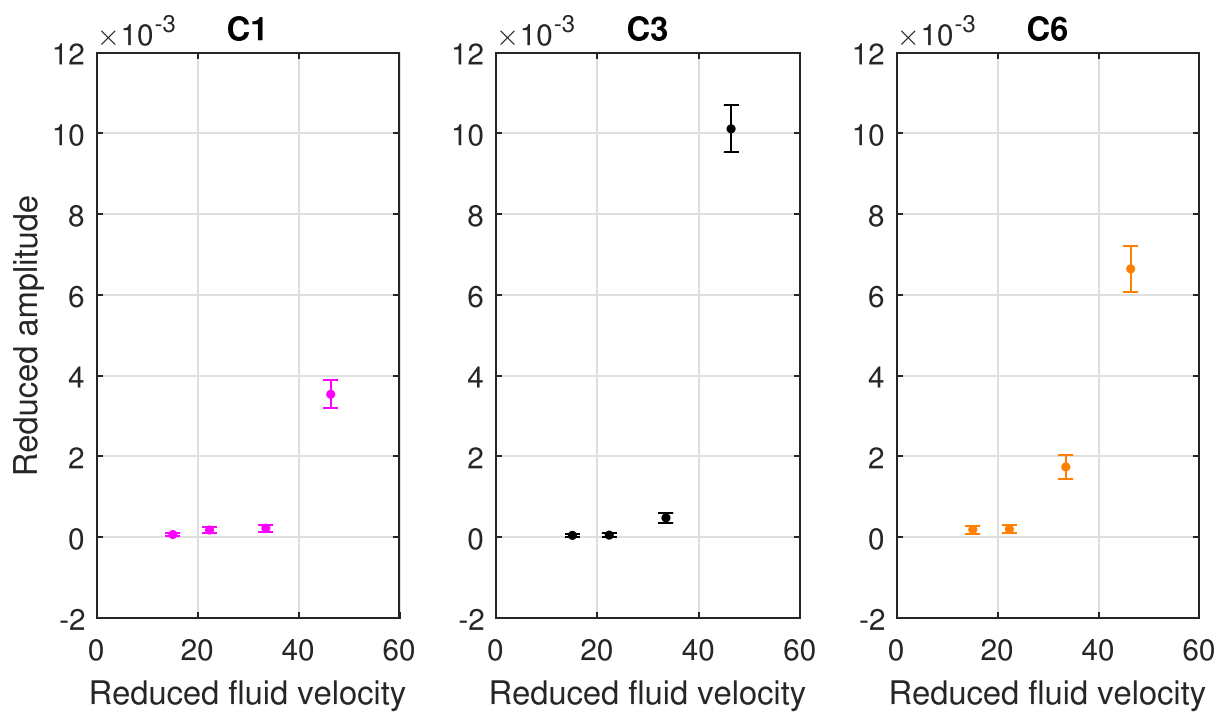

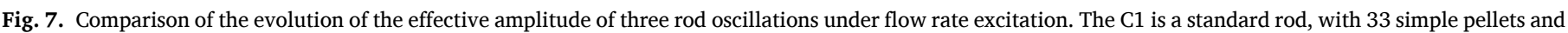

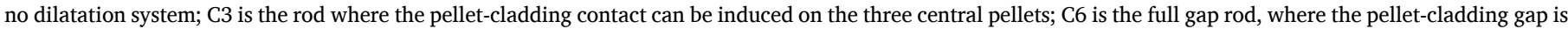
fulfilled with glue as to avoid any internal relative motion. The variance is illustrated with $\mathrm{k}=1$. 
the interaction increased the energy provided to higher modes.

We can thus conclude from the experiment that the pellet-cladding contact can be detected by analyzing the energy dissipated in the structure and we identified a flow rate excitation level at which the local contact can be detected with a good confidence of $90 \%(\mathrm{k}=1.3$ and unilateral risk). The experimental results showed also that the feasibility of the detection is sensitive to the external force applied and the appearance of instabilities.

\section{Conclusions and perspectives}

This work concerned the experimental research to state on the feasibility to detect a local pellet-cladding contact withing a nuclear fuel rod by measuring the evolution of the dynamic response of the rod under passive flow rate excitation. The pellet-cladding contact is a relevant parameter to be detected and eventually characterized on line for studies concerned with the PCI phenomenon. The chosen application frame was the ADELINE irradiation device, where experiences shall be performed to study this phenomenon. The IMPIGRITIA test bench was designed to investigate PCI under the hydraulic excitation representative of ADELINE. IMPIGRITIA was an out-of-pile, low pressure and temperature test, where the $\mathrm{UO}_{2}$ pellets were replaced by Molybdenum, representative of the $\mathrm{UO}_{2}$ mass density. To remotely induce the local closure of the gap, we used a dilatation system and locally replaced some pellets with half-ones.

Contact-less measurements were performed by LDV through a PMMA section. The experimental program was performed in two phases, distinguishing between the global feasibility to detect the pelletcladding contact through the rod free vibrations and the feasibility of the detection under representative flow rate excitation. From the air tests we were able to demonstrate that the local pellet-cladding contact can be detected by analyzing the evolution of the viscous damping ratio of the structure. This difference could be measured with a good confidence in the range of amplitudes $0.2-1 \mathrm{~mm}$. We also showed that the local closure has no measurable effect on the excited frequency of the structure.

During the second experimental phase, we analyzed the effect of the local contact on the effective oscillations amplitude under different flow rate excitation level in the representative range evaluated. We observed that the feasibility of the detection is sensitive to the external force: in the lower range of flow rates, the system barely moves while higher flow rate are affected by flow instability or a modification of the boundary conditions of the system. We were able to identify a flow rate excitation level at which the local contact can be detected with a good confidence of $90 \%(\mathrm{k}=1.3$ and unilateral risk). This shows the interest for further studies to focus on the active excitation of the fuel rod.

IMPIGRITIA constituted the first feasibility test to study the validity of the measurement principle. The design of the device thus underwent several simplifications and adaptations with respect to the ADELINE device: simpler geometry, representative material, out-of-flux study, low temperature and pressure test. The positive results obtained in this experimental campaign allow to foresee further studies in order to state on the feasibility to detect and characterize PCI in ADELINE during irradiation tests in the JHR experimental reactor. In the next studies it is foreseen to test the measurability of the phenomenon using actual $\mathrm{UO}_{2}$ pellets, to study the effect of PCI over longer sections and evaluate the effect of the longer sample holder on the PCI induced attenuation and its measurability.

For application in the nuclear environment of the JHR reactor and in the ADELINE device, a specifically designed measurement method has to be developed. The LDV technology used during the IMPIGRITIA experiment performed really well in the designed operational conditions. However, it is not adapted for in-pile applications in PWR conditions for three main reasons: the LDV uses an Helium-Neon laser with a wavelength of $633 \mathrm{~nm}$ which is strongly attenuated under irradiation (Cheymol et al., 2011), the dimensions of the instrumental set-up are not compatible with the ADELINE loop section and the measurement system is strongly affected by the relative motion of the sensitive measuring head with respect to the target, difficult to be managed under PWR conditions. The use of a modified version of a linear voltage differential transformer to measure the transverse displacement of the fuel rod is under discussion.

\section{CRediT authorship contribution statement}

V. D'Ambrosi: Conceptualization, Data curation, Formal analysis, Investigation, Methodology, Project administration, Software, Visualization, Writing - original draft, Writing - review \& editing. C. Destouches: Conceptualization, Funding acquisition, Methodology, Project administration, Resources, Software, Validation, Writing - review \& editing. G. Ricciardi: Conceptualization, Methodology, Resources, Supervision, Validation, Writing - review \& editing. S. Breaud: Conceptualization, Investigation, Supervision. F. Lebon: Project administration, Resources, Supervision. J.M. Gatt: Conceptualization, Supervision. J. Julien: Supervision, Writing - review \& editing. D. Parrat: Conceptualization, Supervision, Writing - review \& editing.

\section{Declaration of Competing Interest}

The authors declare that they have no known competing financial interests or personal relationships that could have appeared to influence the work reported in this paper.

\section{Acknowledgments}

This work was developed within the framework of the MISTRAL joint research laboratory between Aix-Marseille University, CNRS, Centrale Marseille and CEA. The authors would like to thank all the experts and technicians from CEA and from the Laboratory of Mechanics and Acoustic of Marseille that contributed to this project, for the fruitful collaboration and discussions.

\section{References}

Alberman, A., Roche, M., Couffin, P., Bendotti, S., Moulin, D., Boutfroy, J., 1997. Technique for power ramp tests in the isabelle 1 loop of osiris reactor, Nuclear Eng. Design 168 (1) 293-303.

Axisa, F., 2001. Modélisation des systèmes mécaniques: Vibrations sous écoulements. Hermes Vol. 4

Benhamadouche, S. et al., 2009. CFD estimation of the flow induced vibrations of fuel rod downstream a mixing grid. In: proceeding of the ASME 2009 Pressure vessels and Piping division conference.

Catterou, T., Blanc, V., Ricciardi, G., Bourgeois, S., Cochelin, B., 2020. Non-Linear damping of sodium fast reactor fuel pins: Experimental analysis and numerical modelisation. Nucl. Eng. Des. 364, 110643 https://doi.org/10.1016/j. nucengdes.2020.110643.

Chen, S., Wambsganss, M., 1971. Tentative design guide for calculation the vibration response of flexible cylindrical elements in axial flow, Tech. rep., ANL-ETD-71-07, Argonne National Laboratory.

Chen, S., Wambsganss, M., 1985. Flow-induced vibration of circular cylindrical structures, Tech. rep., ANL-85-51, Argonne National Laboratory.

Chen, Y., Wever, M., 1970. Flow-Induced Vibration in tube bundle heat exchangers with cross and parallel flow. In: ASME Symposium on Flow-Induced Vibration in Heat Exchangers, New York, pp. 57-70.

Cheymol, G., Brichard, B., Villard, J., 2011. Fiber optics for metrology in nuclear research reactors - applications to dimensional measurements. IEEE Trans. Nucl. Sci, 58, 1895-1902.

Clinch, J.M., 1969. Measurements of the wall pressure field at the surface of a smoothwalled pipe containing turbulent water flow. J. Sound Vib. 9, 398-419.

D’Ambrosi, V., Breaud, S., Destouches, C., Ricciardi, G., Lebon, E.S.F., Gatt, J., Parrat, D., 2019. Experimental characterization of PCI impact on vibrating fuel rod under axial turbulent flow representative of JHR irradiation device ADELINE: Set-up conception and measurement method, in: Proceedings of ANIMMA 2019. doi:10.1051/epjconf/ 202022504007.

De Pauw, B., Weijtjens, W., Vanlanduit, S., Van Tichelen, K., Berghmans, F., 2015. Operational modal analysis of flow-induced vibration of nuclear fuel rods in a turbulent axial flow. Nucl. Eng. Des. 284, 19-26. https://doi.org/10.1016/j. nucengdes.2014.11.040.

Ferrari, G., Balasubramanian, P., 2018. Non-linear vibrations of nuclear fuel rods. Nucl. Eng. Des. 338, 269-283. 
J. K. Fink, Thermophysical properties of uranium dioxide 279 1-18.

Gonnier, C., Estrade, J., Bignan, G., Maugard, B., 2017. in: Proceedings of IGORR 2017. Heitz, T., 2017. Nonlinear local behaviours and numerical modeling of damping in civil engineering structures in dynamic, Ph.D. thesis, Universit Paris-Saclay.

Michel, B., Sercombe, J., Nonon, C., Michel, F., Marelle, V., 2013. Simulation of PelletCladding Interaction with the PLEIADES Fuel Performance Software Environment. Nucl. Technol. 182, 124-137.

Païdoussis, M.P., 1969. An experimental study of vibration of flexible cylinders induced by nominally axial flow. Nuclear Sci. Eng. 127-138.

Païdoussis, M.P., 1981. Fluidelastic vibration of cylinder arrays in axial and cross flow: State of the art. J. Sound Vib. 76 (3), 329-360. https://doi.org/10.1016/0022-460X (81)90516-2.

https://www.asn.fr/Professionnels/Installations-nucleaires/Guides-de-l-ASN/Guidesde-1-ASN-n-22-Conception-des-reacteurs-a-eau-sous-pression, online version, 18th July, 2017.
Païdoussis, M.P., 2004. Fluid-Structure Interactions, Slender Structures and Axial Flow, Vol. 2, ELSEVIER.

Park, N., Rhee, H., Park, J.-K., Jeon, S.-Y., Kim, H.-K., 2009. Indirect estimation method of the turbulence induced fluid force spectrum acting on a fuel rod, Nuclear Engineering and Design 239 (7) 1237-1245. doi:10.1016/j.nucengdes.2009.02.020.

Pauw, B.D., Vanlanduit, S., Tichelen, K.V., Geernaert, T., Chah, K., Berghmans, F., 2013. Benchmarking of deformation and vibration measurement techniques for nuclear fuel pins. doi:10.1016/j.measurement.2013.07.003.

Prakash, V., Thirumalai, M., Anandaraj, M., Kumar, P.A., Ramdasu, D., Pandey, G., Padmakumar, G., Anandbabu, C., Kalyanasundaram, P., 2011. Experimental qualification of subassembly design for prototype fast breeder reactor, Nuclear Engineering and Design 241 (8) 3325-3332. doi:10.1016/j.nucengdes.2011.04.040. 